Article

\title{
Structural Behavior of a Multi-Layer Based Microbeam Actuator
}

\author{
Abdulrahman Alofi, Hassen M. Ouakad * and Mohammad Tausiff \\ Department of Mechanical Engineering, King Fahd University of Petroleum and Minerals, Dhahran 31261, \\ Kingdom of Saudi Arabia; amaloufi@kfupm.edu.sa (A.A.); mtausiff@kfupm.edu.sa (M.T.) \\ * Correspondence: houakad@kfupm.edu.sa; Tel.: +966-13-860-3819
}

Academic Editor: Jose Luis Sanchez-Rojas

Received: 17 May 2016; Accepted: 25 August 2016; Published: 31 August 2016

\begin{abstract}
In this paper, the structural behavior of a micro-electromechanical system (MEMS) composed of two electrically coupled parallel clamped-clamped microbeams is investigated. An Euler Bernoulli beam model is considered along with the nonlinear electric actuating force to get the equation of motion governing the structural behavior of the actuator. A reduced-order modeling (ROM) based on the Galerkin expansion technique, while assuming linear undamped mode shapes of a straight fixed-fixed beam as the basis functions, is assumed as a discretization technique of the equations of motion in this investigation. The results showed that the double-microbeam MEMS actuator configuration requires a lower actuation voltage and a lower switching time as compared to the single microbeam actuator. Then, the effects of both microbeams air gap depths were investigated. Finally, the eigenvalue problem was investigated to get the variation of the fundamental natural frequencies of the coupled parallel microbeams with the applied actuating DC load.
\end{abstract}

Keywords: MEMS; actuator; multi-layers; nonlinear structural behavior; electrically-coupled parallel microbeams

\section{Introduction}

Since the 1980s, when micro-electro-mechanical-systems (MEMS) were initially commercialized, the demands for these tiny devices have increased dramatically. This is principally due to their outstanding properties (mechanical, electrical, thermal, etc.) as well as their unique features (small sizes, easiness in fabrication, etc.). While they were used in the beginning mainly as sensors and actuators, they are nowadays designed to be used in many other engineering applications [1]. To cite few, they are being used as pressure sensors [2,3], accelerometers [4,5], microphones in cellphones [6], micro-mirrors in plasma TVs [7], GPS [8] and many other useful applications. Moreover, they are still being continuously explored by scientists and researchers, and these developments will hopefully lead to more useful features and become even more important in life.

In the MEMS community, there are many different types of structures available and the selection depends on the type of the application. For example, clamped-clamped microbeams form one of the basic structures configurations for building MEMS devices. They have various preferred features such as: easiness in the fabrication, high sensitivity, cost effectiveness, etc., make them very attractive in many MEMS-related applications. Furthermore, their fabrication process is somehow trivial and can be done by using the basic bulk and surface micromachining techniques $[9,10]$. Moreover, the natural frequencies of the clamped-clamped microbeam are relatively higher as compared to other microstructures such as cantilever or simply-supported microbeams. This feature is desirable in increasing the sensitivity of the microstructures to be used as Radio Frequency (RF) filters [11], RF switches [12] and resonant sensors [13]. Furthermore, the linear dynamic range of clamped-clamped micro-beams is lower compared to micro-cantilevers [14]. In addition to that, a lot of researches proved 
that the performance of the clamped-clamped microbeam can be improved further. For example, Kacem et al. [15] proved that by tuning the parameters linear behavior can be achieved up to the pull-in point, which could have a great impact on the sensors' resolution. In addition, Kacem et al. [16] showed helpful analysis to enhance the sensors either by avoiding instability branches or gaining some desired features. In another work, Kacem et al. [17] developed a method to improve the frequency stability and the sensing of the resonators. The clamped-clamped microbeam configuration possesses numerous applications and to mention some of them the projection display arrays [18], optical fibers [19] and thermal actuators [20].

Another common configuration of microbeam based MEMS devices is the parallel-plates actuator made from a single movable/flexible electrode. The use of this type of configuration provides an extended range of travel compared to others, however with high power consumption and switching time. These latter specifications are needed in many applications but not for others, where more desirable features and better performing structures are required. One of the suggested alternatives is the use of multi-layers based actuator instead of only one layer (movable electrode) based MEMS actuators.

There are few research groups discussed the applications for the double-microbeam. For example, Afrang et al. [21] suggested the double-microbeam actuator to be used in micro-machined switches application. Since, it has lower actuation voltage without high effect on the switching time. In addition, Chaffey et al. [22] recommended the double cantilever microbeam for the tunable micro-cantilever switches. This is due to the fact that it requires lesser voltage to reach to the pull-in voltage than other single structures. In addition, Samaali et al. [23] assured that the use of double-microbeam helps in reducing the actuation voltage and the static pull in voltage, which makes this structure powerful for RF-MEMS switches. Additionally, Ouakad et al. [24] mentioned that the use of double-microbeam is helpful for applications that require large response. Therefore, it is clear that assuming a double-microbeam, or even multi-layers configuration, may increase the deflection of the microbeam with the same voltage that is provided in a single microbeam. As a result, it might help reducing the power consumption and the switching time for these types of applications where large deformation is needed with minor power consumption as well lower switching time.

It is clear from the afore-summarized literature review that it is important to study the structural behavior of electrically coupled double-microbeams MEMS actuator. More scopes may still be discovered that may open new perspectives of MEMS actuators of interesting features, or even inventing new smart MEMS devices of improved structural and electrical behaviors. This paper will shed light on a simple model of double-microbeam configuration. First, the problem formulation of this system will be discussed, and followed by the reduced-order modeling (ROM) derivation. Then, the static analysis of the system will be presented and a parametric study on the air gap depths will be undertaken. Finally, the eigenvalue problem will be solved to variation of the natural frequencies and the mode shapes of the system will be discussed.

\section{Problem Formulation}

To be able to compare both actuator configurations, we will consider both models for a single-microbeam MEMS based actuator configuration and a double-microbeam configuration are shown in Figure 1a,b, respectively. All shown microbeams in both models are assumed to be clamped-clamped and actuated by a parallel-plates DC bias of amplitude $\left(V_{D C}\right)$ in Volt. For the double microbeam configuration, we consider dielectric layers for each electric field.

Assuming the so-called Newton second law under the assumption of the Euler Bernoulli's shallow-thin beam theory, the governing static equation of motion for the single-microbeam configuration of Figure 1a and its respective boundary conditions can be written as [25]:

$$
E I \frac{d^{4} \hat{w}}{d \hat{x}^{4}}=\left(\frac{E A}{2 L} \int_{0}^{L}\left(\frac{d \hat{w}}{d \hat{x}}\right)^{2} d \hat{x}\right) \frac{d^{2} \hat{w}}{d \hat{x}^{2}}+\frac{\varepsilon_{0} b V_{D C}{ }^{2}}{2(d-\hat{w})^{2}}
$$




$$
\hat{w}(0)=0, \quad \hat{w}(L)=0, \quad \frac{d \hat{w}}{d \hat{x}}(0)=0, \quad \frac{d \hat{w}}{d \hat{x}}(L)=0
$$

where: $E$ is the microbeam Young's modulus of elasticity; $\rho$ is its mass density; $I=(1 / 12) b h^{3}$ is its moment of inertia, where $b$ and $h$ are its respective width and the thickness, respectively; $\hat{w}$ is its static deflection function; $A=b h$ is its cross sectional area; $L$ is its initial length; $\varepsilon_{0}$ is the parallel-plates capacitor dielectric constant; and $d$ is its initial air gap depth.

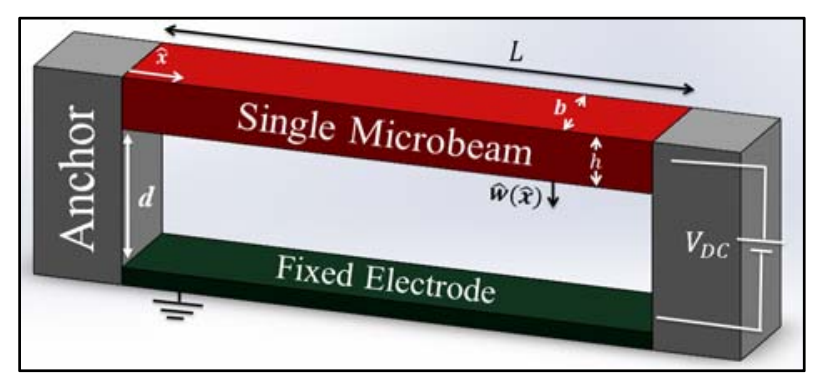

(a)

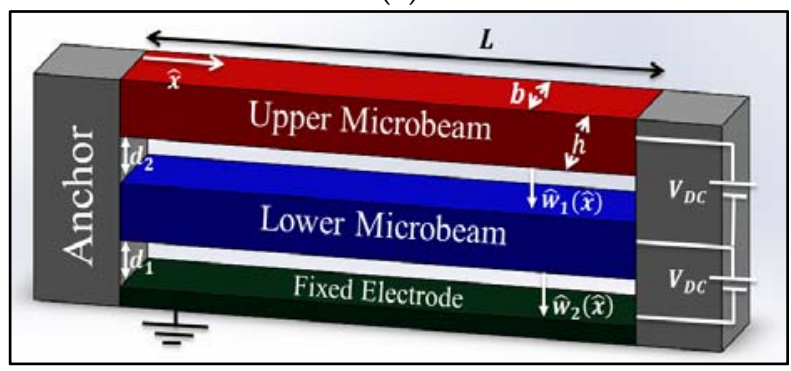

(b)

Figure 1. Schematic of a parallel-plates based DC electrostatic actuator assuming: (a) a single-microbeam; (b) a double-microbeams configuration.

For the double-microbeams configuration shown in Figure 1b, the lower and upper microbeams static equations are respectively given as:

$$
\left\{\begin{array}{l}
\text { Lower Microbeam } \rightarrow E I \frac{d^{4} \hat{w}_{1}}{d \hat{x}^{4}}=\left(\frac{E A}{2 L} \int_{0}^{L}\left(\frac{d \hat{w}_{1}}{d \hat{x}}\right)^{2} d \hat{x}\right) \frac{d^{2} \hat{w}_{1}}{d \hat{x}^{2}}+\frac{\varepsilon_{0} b V_{D C} C^{2}}{2\left(d_{1}-\hat{w}_{1}\right)^{2}}-\frac{\varepsilon_{0} b V_{D C}{ }^{2}}{2\left(d_{2}+\hat{w}_{1}-\hat{w}_{2}\right)^{2}} \\
\text { Upper Microbeam } \rightarrow E I \frac{d^{4} \hat{w}_{2}}{d \hat{x}^{4}}=\left(\frac{E A}{2 L} \int_{0}^{L}\left(\frac{d \hat{w}_{2}}{d \hat{x}}\right)^{2} d \hat{x}\right) \frac{d^{2} \hat{w}_{2}}{d \hat{x}^{2}}+\frac{\varepsilon_{0} b V_{D C}}{2\left(d_{2}+\hat{w}_{1}-\hat{w}_{2}\right)^{2}}
\end{array}\right.
$$

and their respective boundary conditions are set as follows:

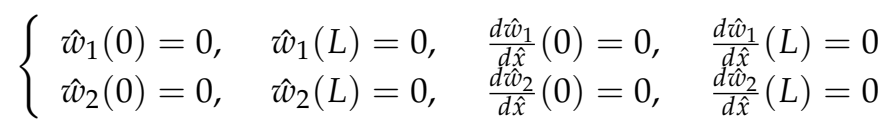

where: $\hat{w}_{i, i=1,2}$ are the static deflections of each microbeam and $d_{i, i=1,2}$ are the two different air gaps for both parallel-plates capacitors.

As it is more convenient to handle calculations and resolutions of equations in the micro-scale in normalized form, we write the above equations of motion in non-dimensional form while assuming the following non-dimensional variables:

$$
w=\frac{\hat{w}}{d}, \quad w_{1}=\frac{\hat{w}_{1}}{d_{1}}, \quad w_{1}=\frac{\hat{w}_{1}}{d_{1}}, \quad w_{2}=\frac{\hat{w}_{2}}{d_{1}}, \quad x=\frac{\hat{x}}{L}
$$

Now, substituting Equation (5) into Equations (1)-(4) result into the following normalized equations of motion and their respective boundary conditions: 
- For the single-microbeam based actuator:

$$
\begin{gathered}
\frac{d^{4} w}{d x^{4}}=\alpha_{1} \Gamma \frac{d^{2} w}{d x^{2}}+\frac{\alpha_{2} V_{D C^{2}}}{(1-w)^{2}} \\
w(0)=0, w(1)=0, \frac{d w}{d x}(0)=0, \frac{d w}{d x}(1)=0,
\end{gathered}
$$

- For the double-microbeams based actuator:

$$
\begin{aligned}
& \left\{\begin{array}{l}
\text { Lower Microbeam } \rightarrow \frac{d^{4} w_{1}}{d x^{4}}=\alpha_{3} \Gamma_{1} \frac{d^{2} w_{1}}{d x^{2}}+\frac{\alpha_{4} V_{D C} C^{2}}{\left(1-w_{1}\right)^{2}}-\frac{\alpha_{4} V_{D C} C^{2}}{\left(d_{2} / d_{1}+w_{1}-w_{2}\right)^{2}} \\
\text { Upper Microbeam } \rightarrow \frac{d^{4} w_{2}}{d x^{4}}=\alpha_{3} \Gamma_{2} \frac{d^{2} w_{2}}{d x^{2}}+\frac{\alpha_{4} V_{D C}}{\left(d_{2} / d_{1}+w_{1}-w_{2}\right)^{2}}
\end{array}\right. \\
& \left\{\begin{array}{cccc}
w_{1}(0)=0, & w_{1}(1)=0, & \frac{d w_{1}}{d x}(0)=0, & \frac{d w_{1}}{d x}(1)=0, \\
w_{2}(0)=0, & w_{2}(1)=0, & \frac{d w_{2}}{d x}(0)=0, & \frac{d w_{2}}{d x}(1)=0,
\end{array}\right.
\end{aligned}
$$

where the nondimensional parameters assumed in Equations (6)-(9) are defined as follows: $\alpha_{1}=6\left(\frac{d}{h}\right)^{2}, \alpha_{2}=\frac{6 \varepsilon_{0} L^{4}}{E h^{3} d^{3}}, \alpha_{3}=6\left(\frac{d_{1}}{h}\right)^{2}, \alpha_{4}=\frac{6 \varepsilon_{0} L^{4}}{E h^{3} d_{1}{ }^{3}}, \Gamma=\int_{0}^{1}\left(\frac{d w}{d x}\right)^{2} d x, \Gamma_{i, i=1,2}=\int_{0}^{1}\left(\frac{d w_{i}}{d x}\right)^{2} d x$.

\section{Reduced-Order Model (ROM)}

To solve the nonlinear differential equations governing the structural behavior of the above described MEMS actuators, various methods can be assumed such as the Finite-Element Method [26], the Finite-Difference Method [27], Differential-Quadrature Method [27], the Shooting Method [28,29], etc., which are considered to be computationally expensive and in some cases unstable since some rely on initial guesses. Another powerful technique is the so-called Galerkin expansion discretization which is mainly used to derive Reduced-Order Models (ROM) from distributed (continuous) systems. This method is a well-used technique in the literature of MEMS devices [30].

As a result, in order to get the ROM, the previous equations, i.e., Equations (6)-(9), will be discretized using Galerkin method and this can be done by expanding the static deflections of the assumed microbeams as:

$$
w(x)=\sum_{i=1}^{N} k_{i} \phi_{i}(x), \quad w_{1}(x)=\sum_{i=1}^{N} f_{i} \phi_{i}(x), \quad w_{2}(x)=\sum_{i=1}^{N} g_{i} \phi_{i}(x)
$$

where the coefficients $k_{i}, f_{i}$, and $g_{i}$ are time-independent constants and $\phi_{i}(x)$ are trial functions assumed to be the linear mode shapes of a clamped-clamped beam. To solve for the time-independent unknowns constants $k_{i}, f_{i}$, and $g_{i}$, it is essential to substitute Equation (10) into Equations (6)-(9), then multiply the outcome by $\phi_{j}(x)$, and finally integrate the outcome from $x=0$ to $x=1$ while using the orthogonality of the mode shapes functions of a clamped-clamped beam. Following the previous procedure, we get the following reduced-order modeling equations bot both assumed actuators as follows:

- For the single-microbeam based actuator:

$$
\begin{aligned}
& \int_{x=0}^{1} \phi_{j}(x) \sum_{i=1}^{N} k_{i} \phi_{i}^{i v}(x) d x=\int_{x=0}^{1} \phi_{j}(x) \frac{\alpha_{2} V_{D C}^{2}}{\left(1-\sum_{i=1}^{N} k_{i} \phi_{i}(x)\right)^{2}} d x \\
& +\int_{x=0}^{1} \phi_{j}(x)\left(\alpha_{1} \int_{0}^{1}\left(\sum_{i=1}^{N} k_{i} \phi_{i}^{\prime}(x)\right)^{2} d x\right) \sum_{i=1}^{N} k_{i} \phi_{i}^{\prime \prime}(x) d x
\end{aligned}
$$


- For the double-microbeams based actuator:

$$
\begin{aligned}
& \text { Lower Microbeam } \rightarrow \int_{x=0}^{1} \phi_{j}(x) \sum_{i=1}^{N} f_{i} \phi_{i}^{i v}(x) d x=\int_{x=0}^{1} \phi_{j}\left(\left(\alpha_{3} \int_{0}^{1}\left(\sum_{i=1}^{N} f_{i} \phi_{i}^{\prime}(x)\right)^{2} d x\right) \sum_{i=1}^{N} f_{i} \phi_{i}^{\prime \prime}(x)\right) d x+ \\
& \alpha_{4} V_{D C}^{2}\left(\int_{x=0}^{1} \frac{\phi_{j}(x)}{\left(1-\sum_{i=1}^{N} f_{i} \phi_{i}(x)\right)^{2}} d x-\int_{x=0}^{1} \frac{\phi_{j}(x)}{\left(d_{2} / d_{1}+\sum_{i=1}^{N} f_{i} \phi_{i}(x)-\sum_{i=1}^{N} g_{i} \phi_{i}(x)\right)^{2}} d x\right) \\
& \text { Upper Microbeam } \rightarrow \int_{x=0}^{1} \phi_{j}(x) \sum_{i=1}^{N} g_{i} \phi_{i}^{i v}(x) d x=\alpha_{3} \int_{x=0}^{1} \phi_{j}\left(\left(\int_{0}^{1}\left(\sum_{i=1}^{N} g_{i} \phi_{i}^{\prime}(x)\right)^{2} d x\right) \sum_{i=1}^{N} g_{i} \phi_{i}^{\prime \prime}(x)\right) d x+ \\
& +\alpha_{4} V_{D C}^{2} \int_{x=0}^{1}\left(\frac{\phi_{j}(x)}{\left(d_{2} / d_{1}+\sum_{i=1}^{N} f_{i} \phi_{i}(x)-\sum_{i=1}^{N} g_{i} \phi_{i}(x)\right)^{2}}\right) d x
\end{aligned}
$$

\section{Static Analysis}

In this section, the static analysis for both single and double-microbeams configuration is carried out using the earlier derived ROM. The main problem with this approach is that the distributed electrostatic force comes in an integral form in the resulting ROM equations, and consequently this integral form is not easy to deal analytically due to nonlinearities arising from its denominator. As an attempt to overcome this challenge, some groups [31,32] used Taylor-series expansion procedure, which brings the nonlinearity to the numerator of the electrostatic force and hence simplifies the calculation of nonlinear ROM integrals. Others suggested multiplying the resultant equation of motion by the electrostatic force denominator and hence the evaluation of the integral in the ROM equation will not be problematic anymore [25]. We adopted to use this latter approach which is numerically stable and more convenient in dealing with nonlinear forcing terms.

In order to ascertain the convergence of the implemented ROM, we start the calculation of the microbeams' deflections with only one mode in the ROM. For the single microbeam, we solve for the $k_{i}$ in Equation (11), whereas for double microbeam we solve for $f_{i}$, and $g_{i}$ in the coupled Equation $(12 \mathrm{a}, \mathrm{b})$, and this can be done by several methods like the harmonic balance method coupled with the asymptotic numerical method [33,34], which enables the capture of stable and unstable branches or using the so-called Newton's method. We adopted the latter approach by using the command FindRoot in Mathematica software. Then increase the number of assumed modes in the ROM by one. The previous steps are to be repeated until the maximum deflection variation with $V_{D C}$ for both microbeams is converging. The maximum deflection of the upper microbeam is presented versus the applied DC voltage in Figure 2 for the case study of Table 1. It can be noted from the graph that when the number of modes is increased the solution is varying slightly, until convergence is reached at almost three modes in the ROM.

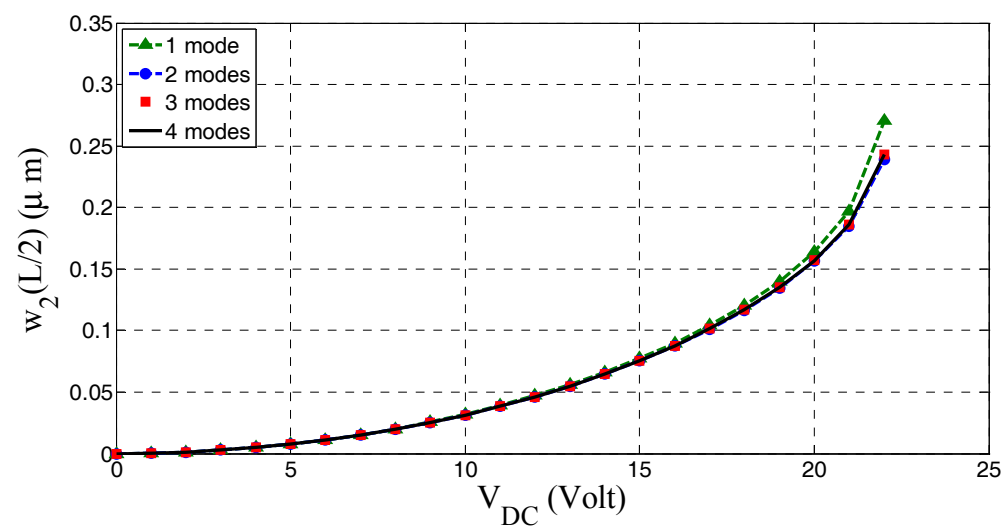

Figure 2. Convergence of the upper microbeam maximum static deflection assuming the case study of Table 1. 
Table 1. Selected case study of a double-microbeams based actuator.

\begin{tabular}{cccc}
\hline Parameter & Value & Parameter & Value \\
\hline Beam Length $(L)$ & $150 \mu \mathrm{m}$ & Effective young's modulus $(E)$ & $124 \mathrm{GPa}$ \\
Beam thickness $(h)$ & $1.0 \mu \mathrm{m}$ & Density $(\rho)$ & $2332 \mathrm{~kg} / \mathrm{m}^{3}$ \\
Beam width $(b)$ & $4.0 \mu \mathrm{m}$ & Air gap depth $\left(d_{1}\right.$ and $\left.d_{2}\right)$ & $1.25 \mu \mathrm{m}$ \\
\hline
\end{tabular}

Next, a parametric study is carried out to investigate the effect of changing the air gaps between the movable microbeams on their static profiles. Three different sets of air gaps are assumed, which are: 1.0, 1.25 and $1.5 \mu \mathrm{m}$ and they were all restricted by the following condition (to be able to compare with the single-beam based actuator with initial gap of $4 \mu \mathrm{m}$ ):

$$
d_{1}+d_{2}+h=d=4 \mu \mathrm{m}
$$

Accordingly, three different cases were considered, which are:

- $\quad$ Case 1: $d_{1}=d_{2}$ (where both initial gaps are set equal to $1.25 \mu \mathrm{m}$ )

- $\quad$ Case 2: $d_{1}>d_{2}$ (where $d_{1}=1.5 \mu \mathrm{m}$ and $d_{2}=1.0 \mu \mathrm{m}$ )

- $\quad$ Case 3: $d_{1}<d_{2}$ (where $d_{1}=1.0 \mu \mathrm{m}$ and $d_{2}=1.5 \mu \mathrm{m}$ )

\subsection{Case $1\left(d_{1}=d_{2}\right)$}

For this first case, the maximum static deflection versus the applied DC voltage for both microbeams (upper and lower) is presented in Figure 3. It is clear from the figure that, the upper microbeam is deflected downward (positive value in Figure 3), which seems to be reasonable, since the upper microbeam is affected by the force from the lower microbeam, which pulls it downward. In contrast, the lower microbeam is deflected upward (negative value in Figure 3), which means that the force which results from the potential between the two microbeams is higher than the force from the fixed (stationary) electrode. In addition, the downward deflection for the upper microbeam is higher than the lower microbeam and it reaches pull-in first, which is about 23 Volt.

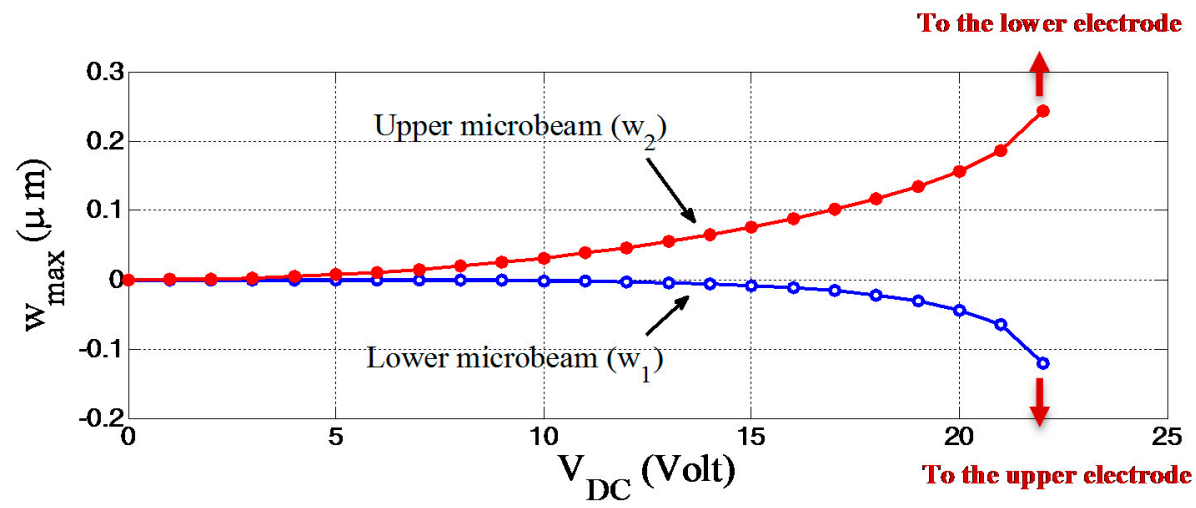

Figure 3. Variation of the maximum static deflection with the DC voltage for the lower and upper microbeams and for the case when $d_{1}=d_{2}$.

\subsection{Case $2\left(d_{1}>d_{2}\right)$}

In this particular case, the behavior of the maximum static deflection for both microbeams is similar to the previous case, as displayed in Figure 4. Again, the upper microbeam reaches the pull-in instability first at about 15 Volt. As compared to the previous case, the pull-in voltage is reduced and more deflection will occur in this case if similar voltages are assumed. 


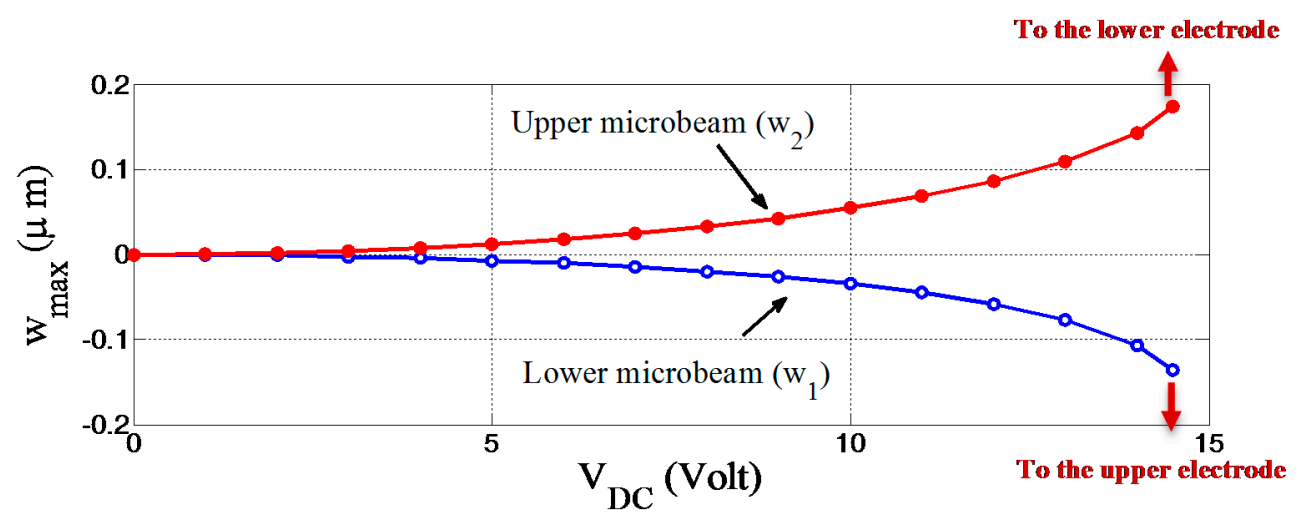

Figure 4. Variation of the maximum static deflection with the DC voltage for the lower and upper microbeams and for the case when $d_{1}>d_{2}$.

\subsection{Case $3\left(d_{1}<d_{2}\right)$}

In this latter case, when calculating and then displaying the maximum static deflection versus the applied voltage, as shown in Figure 5, we can identify some interesting and different outcomes in comparison to the two previously investigated cases. To mention a few, the lower microbeam, in this particular case, is deflected downward, which means the force resulting from the fixed electrode is higher than the force exerted by the upper microbeam. Furthermore, the magnitude of the deflection for the lower microbeam is higher than the upper and it reaches the pull-in instability first at about 23 Volt. Moreover, when the lower microbeam undergoes the pull-in instability (losses its stiffness) at around 23 Volt, the upper microbeam is still safe and possess some stiffness which states that it can be still used as actuator (with more range of travel).

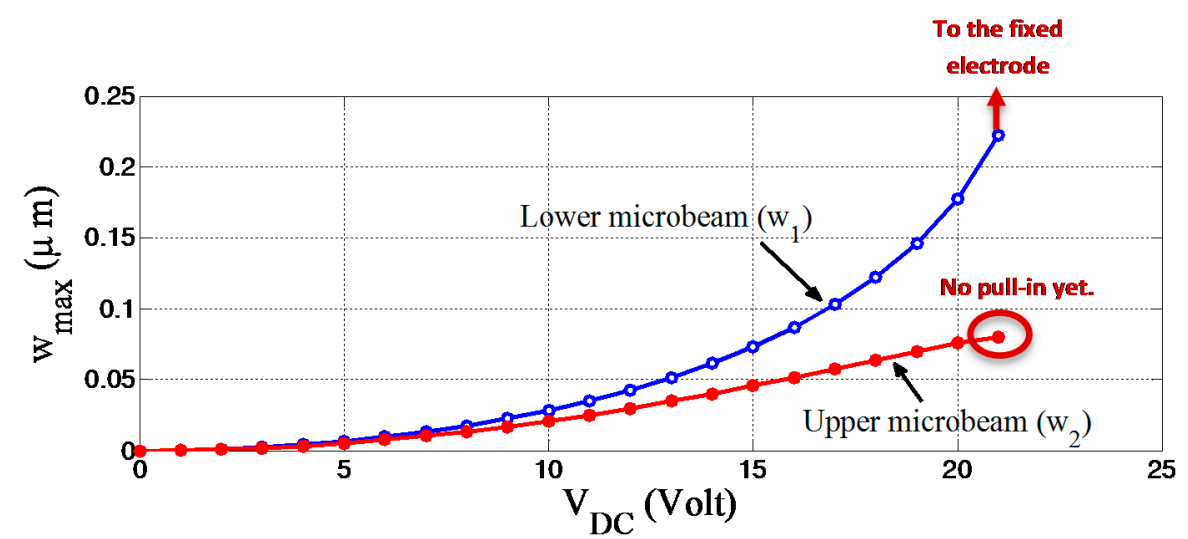

Figure 5. Variation of the maximum static deflection with the DC voltage for the lower and upper microbeams and for the case when $d_{1}<d_{2}$.

Finally, a comparison between the static deflection for the upper microbeam, when considering all three investigated cases as well as the case considering only single microbeam is presented in Figures 6 and 7. Therefore, in all of the three investigated cases more deflection will be provided at the same voltage if assuming the double-microbeams rather than a single-microbeam arrangement. However, the pull-in voltage is reduced by a significant amount, since for the single-microbeam shape, the pull-in voltage is about 236 Volt. Hence, more stroke with lower power consumption. Table 2 compares the pull-in voltage for the three cases with the single microbeam. 


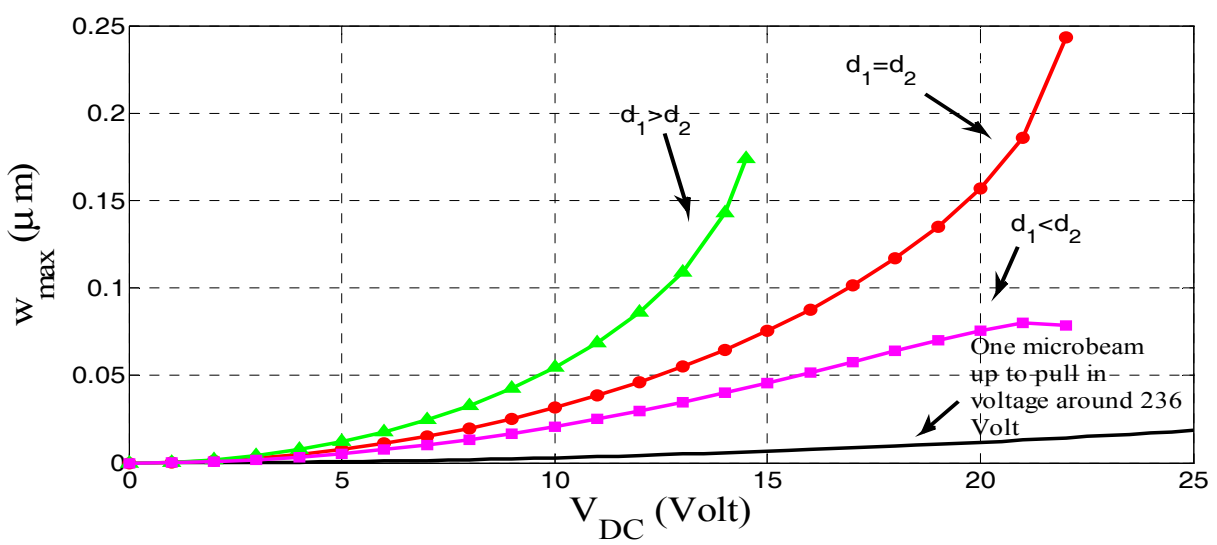

Figure 6. Comparison between the variations of the upper microbeam maximum static deflections with the DC voltage for all three cases with the single-microbeam case.

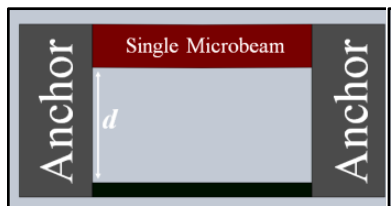

(a)

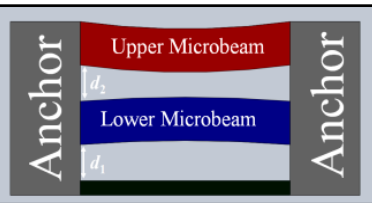

(b)

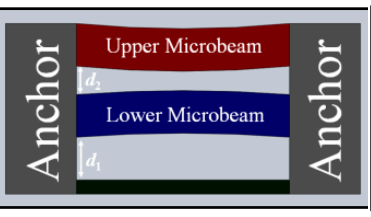

(c)

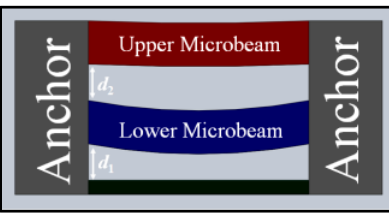

(d)

Figure 7. Comparison between the static profiles of (a) the single-microbeam configuration at $V_{D C}=23$ Volt with the double microbeam configuration just before the pull-in voltage for the case of: (b) $d_{1}=d_{2} ;$ (c) $d_{1}>d_{2} ;$ (d) $d_{1}<d_{2}$ (Note: the length (L) in the above sketches is not to the scale).

Table 2. Comparison of pull-in voltage of all cases shown in Figure 7.

\begin{tabular}{lll}
\hline Assumed Structure & Pull-in Voltage \\
\hline \multirow{3}{*}{ Double-microbeams } & Case $d_{1}=d_{2}$ & 23 Volt \\
\cline { 2 - 3 } & Case $d_{1}>d_{2}$ & 15 Volt \\
\cline { 2 - 3 } & Case $d_{1}<d_{2}$ & 23 Volt \\
\hline Single-microbeam & & 236 Volt \\
\hline
\end{tabular}

An insight into Figure 6 reveals an interesting behavior. While, in all of the previous configurations for all points, increasing the voltage makes the upper microbeam approach the pull-in voltage faster, it can be seen that for the last point in configuration of $d_{1}<d_{2}$ there is no such tendency. This strange behavior may be because the lower microbeam in this case is very close to the fixed electrode, and so the distance between the two microbeams will be higher. This makes the force between the two microbeams lower, and hence the upper microbeam will be far from the pull-in instability allowing it to still vibrate safely.

\section{Natural Frequencies and Mode Shapes}

In order to get the natural frequencies of the above considered MEMS based actuators, the inertia terms $\left(w_{, t t}, w_{1, t t}\right.$ and $\left.w_{2, t t}\right)$ will be included in the right hand side of both Equations (6) and (8), respectively. The subscript, $t$ stands for the second time derivative with respect to the time variable $t$. Thus, Equations (6) and (8) will be respectively adjusted to include these inertia effects as follows:

$$
\text { Single Microbeam } \rightarrow \frac{\partial^{4} w}{\partial x^{4}}+\frac{\partial^{2} w}{\partial t^{2}}=\alpha_{1} \Gamma \frac{\partial^{2} w}{\partial x^{2}}+\frac{\alpha_{2} V_{D C^{2}}}{(1-w)^{2}}
$$




$$
\left\{\begin{array}{l}
\text { Lower Microbeam } \rightarrow \frac{\partial^{4} w_{1}}{\partial x^{4}}+\frac{\partial^{2} w_{1}}{\partial t^{2}}=\alpha_{3} \Gamma_{1} \frac{\partial^{2} w_{1}}{\partial x^{2}}+\frac{\alpha_{4} V_{D C}}{\left(1-w_{1}\right)^{2}}-\frac{\alpha_{4} V_{D C^{2}}}{\left(d_{2} / d_{1}+w_{1}-w_{2}\right)^{2}} \\
\text { Upper Microbeam } \rightarrow \frac{\partial^{4} w_{2}}{\partial x^{4}}+\frac{\partial^{2} w_{2}}{\partial t^{2}}=\alpha_{3} \Gamma_{2} \frac{\partial^{2} w_{2}}{\partial x^{2}}+\frac{\alpha_{4} V_{D C}{ }^{2}}{\left(d_{2} / d_{1}+w_{1}-w_{2}\right)^{2}}
\end{array}\right.
$$

Subsequently, the deflections for single-microbeam based actuator as well as the lower and upper microbeams for the case of double-microbeams based actuator will be again discretized using the Galerkin expansion technique as follows:

$$
\begin{gathered}
w(x, t)=\sum_{i=1}^{N}\left(k_{i}+\beta(t)\right) \phi_{i}(x) \\
\left\{\begin{array}{l}
w_{1}(x, t)=\sum_{i=1}^{N}\left(f_{i}+\mu_{i}(t)\right) \phi_{i}(x) \\
w_{2}(x, t)=\sum_{i=1}^{N}\left(g_{i}+v_{i}(t)\right) \phi_{i}(x)
\end{array}\right.
\end{gathered}
$$

where: $k_{i}, f_{i}$ and $g_{i}$ are the time-independents unknown constants that were calculated in the static analysis part and $\beta(t), \mu_{i}(t)$ and $v_{i}(t)$ are time-dependent unknown functions. Details about the derivation of the single-microbeam based actuator can found in [25]. We will focus next on the derivation of the equations governing the eigenvalue problem of the double-microbeams based actuator. Subsequently, and since we are in the process of developing the linear eigenvalue problem, the nonlinear electrostatic force terms in the derived ROM equations are to be linearized using the so-called Taylor series expansion. Therefore, neglecting all of the higher order terms to obtain:

$$
M\left(f_{i}, g_{i}\right) \overline{\dot{\eta}}=J\left(f_{i}, g_{i}\right) \bar{\eta}
$$

where, $J\left(f_{i}, g_{i}\right)$ is known as the Jacobian matrix of the linearized eigenvalue problem. The natural frequencies of the beam for a given voltage can be obtained by taking the square roots of the eigenvalues of $M^{-1} J$ and the corresponding eigenvectors will be the respective mode shapes.

The selected parameters are the same we assumed in the static analysis for the double-microbeams arrangement, which is shown in Table 1 with the case of $d_{1}=d_{2}$. The natural frequencies were obtained by using three symmetric modes and were displayed versus the applied voltage as shown in Figure 8 . The obtained results indicate that all of the higher-order natural frequencies are insensitive with the applied voltage, with the exception of the fundamental one (lower frequency). The fundamental natural frequency starts at its maximum position (when no electrical load is applied) and then decreases gradually with an increase in the applied voltage until it gets close to the pull-in voltage, at which point it drops sharply to zero. In addition, it can be noted that each odd natural frequency, when paired with the consecutive one are the same, except for the fundamental frequency, especially at higher applied DC voltages.

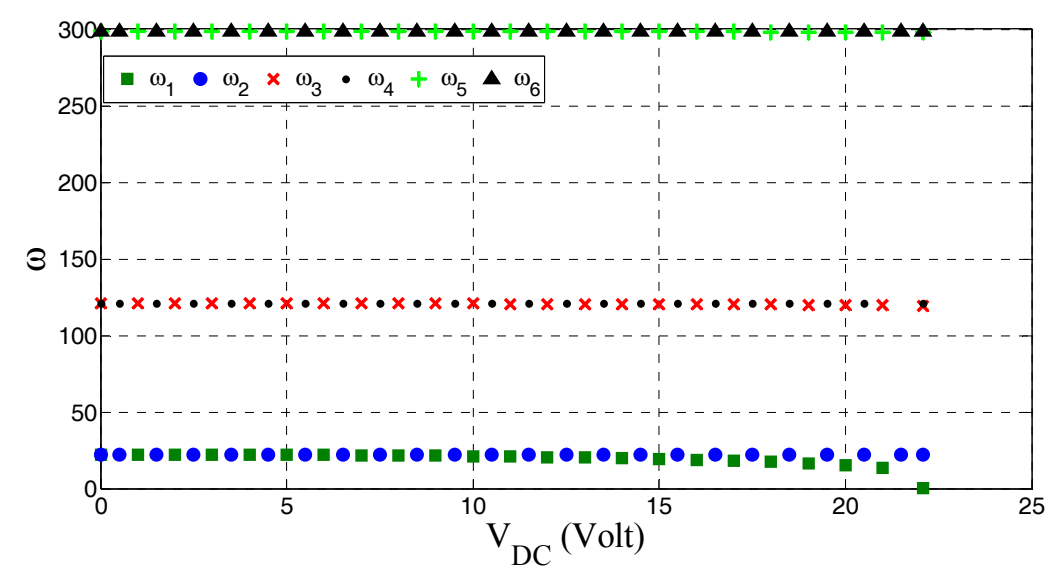

Figure 8. Variation of the first six natural frequencies with the applied DC voltage for the case of the double-microbeams based actuator and for $d_{1}=d_{2}$. 
A comparison between the fundamental natural frequencies among the three cases considered in the static section is shown in Figure 9. The results here seem to follow the static analysis outcomes, since in the case with the lowest pull-in voltage the fundamental frequency reaches zero first (when: $\left.d_{1}>d_{2}\right)$. Moreover, the other two cases reach the pull-in voltage at about the same value (23 Volt) and accordingly their fundamental natural frequency drops to zero or close to it.

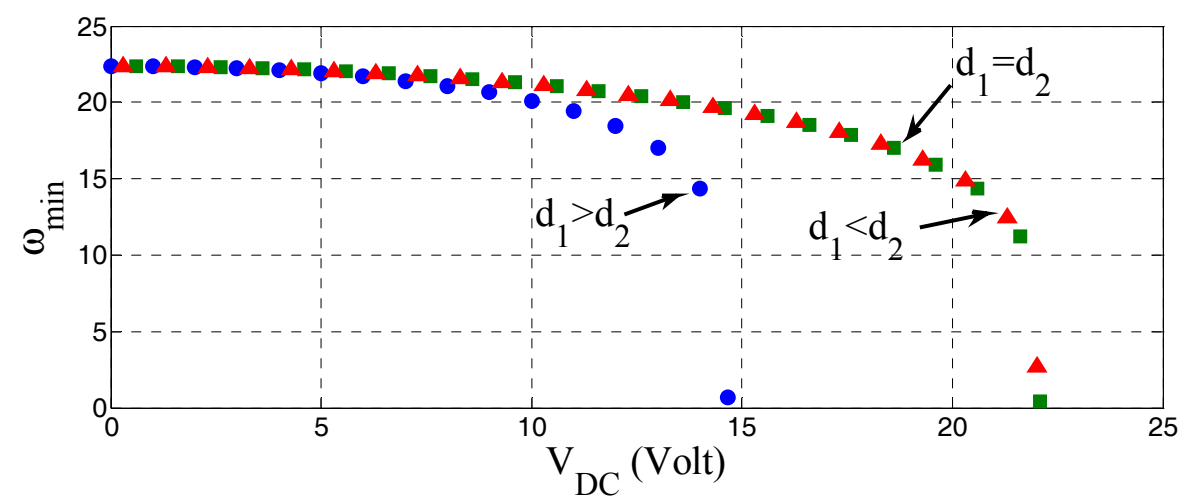

Figure 9. Variation of the fundamental natural frequencies with the applied DC voltage for the case of the double-microbeams based actuator and for three different cases of the microbeams initial air gaps.

The first and second coupled mode shapes for the case of $d_{1}=d_{2}$ at $V_{D C}=2$ Volt are presented in Figures 10 and 11, respectively. The results show that for the first mode shape $\phi_{1}$ the coupled modes $\left(\phi_{11}\right.$ and $\left.\phi_{12}\right)$ are opposite to each other, sharing an out-of-phase motion. Conversely, for the second mode shape $\left(\phi_{2}\right)$ the two coupled modes $\left(\phi_{21}\right.$ and $\left.\phi_{22}\right)$ have almost the same magnitude as the first in an absolute value and both of them share an in-phase-motion.

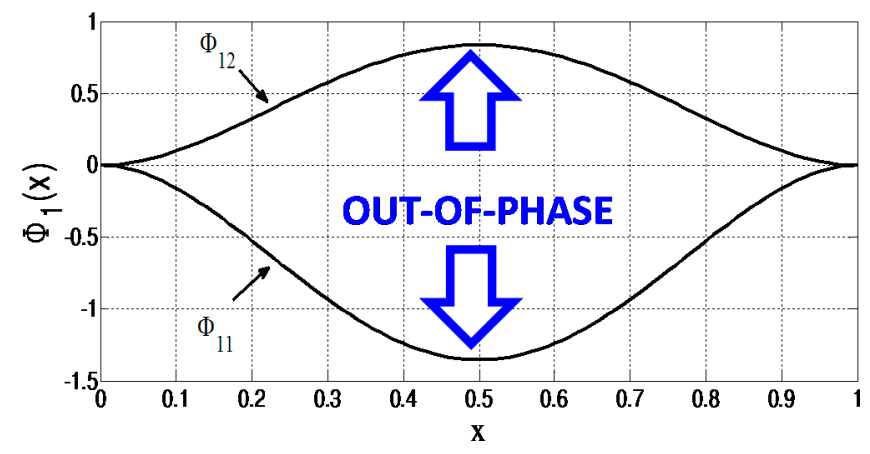

Figure 10. The first coupled mode shape of the double-microbeams case assuming $V_{D C}=2$ Volt.

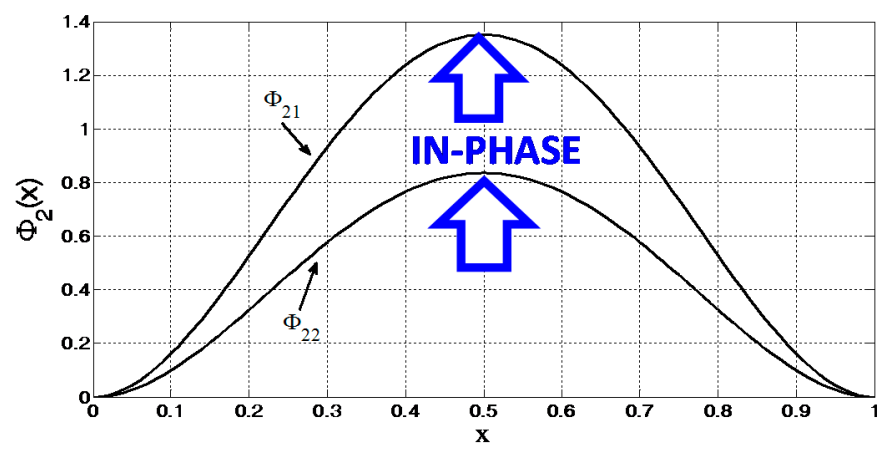

Figure 11. The second coupled mode shape of the double-microbeams case assuming $V_{D C}=2$ Volt. 


\section{Conclusions}

In conclusion, the use of double-microbeams configuration was shown to be useful for applications necessitating low actuation and pull-in voltage, and large deflections (larger stroke). The effects of changing the air gap depths on the double-microbeam configuration revealed interesting structural bending profiles for both the upper and lower electrically coupled microbeams. For example, both microbeams could be directed down to the fixed electrode or the upper microbeam could be directed down while the lower is up. In addition, the pull-in voltage was shown to be different for the different assumed and different air gaps cases. This can be achieved either from the lower microbeam as it reaches the fixed electrode, the lower microbeam as it sticks with the upper microbeam or the upper microbeam as it pulls toward the lower microbeam.

Acknowledgments: Support offered by King Fahd University of Petroleum and Minerals (KFUPM) through its Deanship of Scientific Research (DSR) is extremely acknowledged by all authors.

Author Contributions: Hassen M. Ouakad suggested the idea and model, performed the equations derivation, directed the work and assisted along with all the authors in writing the manuscript. Abdulrahman Alofi automated the coding of the equation, derived the reduced-order model and, simulated the numerical results and drafted the manuscript. Mohammad Tausif assisted in the reduced-order modeling and in the manuscript preparation.

Conflicts of Interest: The authors declare no potential conflicts of interest with respect to the research, authorship, and/or publication of this article.

\section{References}

1. Marek, J.; Hoefflinger, B.; Gomez, U.-M. MEMS-Micro-Electromechanical Sensors for the Internet of Everything. In CHIPS 2020 VOL. 2; Springer: Cham, Switzerland, 2016; pp. 221-229.

2. Lee, J.-S.; Yoo, E.-S.; Park, C.-H.; An, J.-E.; Park, C.G.; Song, J.W. Development of a piezoresistive MEMS pressure sensor for a precision air data module. In Proceedings of the 2014 14th International Conference on Control, Automation and Systems (ICCAS), Kintex, Korea, 22-25 October 2014; pp. 874-878.

3. Abeysinghe, D.C.; Dasgupta, S.; Boyd, J.T.; Jackson, H.E. A novel MEMS pressure sensor fabricated on an optical fiber. IEEE Photonics Technol. Lett. 2011, 13, 993-995. [CrossRef]

4. Gonseth, S.; Rudolf, F.; Eichenberger, C.; Durrant, D.; Airey, P. Miniaturized high-performance MEMS accelerometer detector. CEAS Space J. 2015, 7, 1-8. [CrossRef]

5. Luczak, S.; Oleksiuk, W.; Bodnicki, M. Sensing tilt with MEMS accelerometers. IEEE Sens. J. 2006, 6, 1669-1675. [CrossRef]

6. Loeppert, P.V.; Lee, S.B. SiSonic ${ }^{\mathrm{TM}}$ —The first commercialized MEMS microphone. In Proceedings of the Solid-State Sensors, Actuators, and Microsystems Workshop, Hilton Head, SC, USA, 6-9 June 2006; pp. 27-30.

7. Hopper, D.G. Creation and transition of digital light processing technology to defense applications. Proc. SPIE 2005, 5801, 113-124.

8. Brown, A.K. GPS/INS uses low-cost MEMS IMU. IEEE Aerosp. Electron. Syst. Mag. 2005, 20, 3-10. [CrossRef]

9. Kurhekar, A.S.; Apte, P.; Duttagupta, S. Design and Fabrication of Bulk Micro-machined, High Resilience, High-Q, High Tilt Angle, Low Driving Voltage, Flexure Beam Micro-mirrors on Mono-crystalline Silicon. Silicon 2015, 8, 1-14. [CrossRef]

10. Lee, S.; Park, S.; Cho, D.-I. The surface/bulk micromachining (SBM) process: A new method for fabricating released MEMS in single crystal silicon. J. Microelectromech. Syst. 1999, 8, 409-416.

11. Umesh, G.; Nayak, B.; Deshpande, A. Design and Analysis of a Capacitively-Coupled Clamped-Clamped MEMS Resonator. In Proceedings of the 2014 International Conference on Computational Intelligence and Communication Networks (CICN), Bhopal, India, 14-16 November 2014; pp. 155-161.

12. Mulloni, V.; Solazzi, F.; Resta, G.; Giacomozzi, F.; Margesin, B. RF-MEMS switch design optimization for long-term reliability. Analog Integr. Circ. S. 2014, 78, 323-332. [CrossRef]

13. Younis, M.I.; Alsaleem, F.; Jordy, D. The response of clamped-clamped microbeams under mechanical shock. Int. J. Non Linear Mech. 2007, 42, 643-657. [CrossRef]

14. Kacem, N.; Arcamone, J.; Perez-Murano, F.; Hentz, S. Dynamic range enhancement of nonlinear nanomechanical resonant cantilevers for highly sensitive NEMS gas/mass sensor applications. J. Micromech. Microeng. 2010, 20, 045023. [CrossRef] 
15. Kacem, N.; Hentz, S.; Pinto, D.; Reig, B.; Nguyen, V. Nonlinear dynamics of nanomechanical beam resonators: Improving the performance of NEMS-based sensors. Nanotechnology 2009, 20, 275501. [CrossRef] [PubMed]

16. Kacem, N.; Hentz, S. Bifurcation topology tuning of a mixed behavior in nonlinear micromechanical resonators. Appl. Phys. Lett. 2009, 95, 183104. [CrossRef]

17. Kacem, N.; Baguet, S.; Duraffourg, L.; Jourdan, G.; Dufour, R.; Hentz, S. Overcoming limitations of nanomechanical resonators with simultaneous resonances. Appl. Phys. Lett. 2015, 107, 073105. [CrossRef]

18. Yu, Y.-T.; Yuan, W.-Z.; Qiao, D.-Y. Electromechanical characterization of a new micro programmable blazed grating by laser Doppler vibrometry. Microsyst. Technol. 2009, 15, 853-858. [CrossRef]

19. Zook, J.D.; Burns, D.W.; Herb, W.R.; Guckel, H.; Kang, J.-W.; Ahn, Y. Optically excited self-resonant microbeams. Sens. Actuators A Phys. 1996, 52, 92-98. [CrossRef]

20. Shamshirsaz, M.; Asgari, M. Polysilicon micro beams buckling with temperature-dependent properties. Microsyst. Technol. 2008, 14, 957-961. [CrossRef]

21. Afrang, S.; Abbaspour-Sani, E. A low voltage MEMS structure for RF capacitive switches. Prog. Electromagn. Res. 2006, 65, 157-167. [CrossRef]

22. Chaffey, J.P.; Austin, M. Analytical modeling of the electromechanical coupling of cantilever beams. Proc. SPIE 2002, 4935, 86-93.

23. Samaali, H.; Najar, F.; Choura, S.; Nayfeh, A.H.; Masmoudi, M. A double microbeam MEMS ohmic switch for RF-applications with low actuation voltage. Nonlinear Dyn. 2011, 63, 719-734. [CrossRef]

24. Ouakad, H.M.; Hawwa, M.A.; Al-Qahtani, H.M. Modeling the Electrostatic Deflection of a MEMS Multilayers Based Actuator. Math. Probl. Eng. 2013, 2013. [CrossRef]

25. Younis, M.; Abdel-Rahman, E.M.; Nayfeh, A. A reduced-order model for electrically actuated microbeam-based MEMS. J. Microelectromech. Syst. 2003, 12, 672-680. [CrossRef]

26. Hung, E.S.; Senturia, S.D. Generating efficient dynamical models for microelectromechanical systems from a few finite-element simulation runs. J. Microelectromech. Syst. 1999, 8, 280-289. [CrossRef]

27. Najar, F.; Choura, S.; Abdel-Rahman, E.M.; El-Borgi, S.; Nayfeh, A. Dynamic analysis of variable-geometry electrostatic microactuators. J. Micromech. Microeng. 2006, 16, 2449. [CrossRef]

28. Abdel-Rahman, E.M.; Younis, M.I.; Nayfeh, A.H. Characterization of the mechanical behavior of an electrically actuated microbeam. J. Micromech. Microeng. 2002, 12, 759. [CrossRef]

29. Ouakad, H.M.; Younis, M.I. Modeling and simulations of collapse instabilities of microbeams due to capillary forces. In Proceedings of the ASME 2008 International Mechanical Engineering Congress and Exposition, Boston, MA, USA, 2-6 November 2008; pp. 187-195.

30. Younis, M.I. MEMS Linear and Nonlinear Statics and Dynamics; Springer Science \& Business Media: New York, NY, USA, 2011; Volume 20.

31. Krylov, S.; Ilic, B.R.; Lulinsky, S. Bistability of curved microbeams actuated by fringing electrostatic fields. Nonlinear Dyn. 2011, 66, 403-426. [CrossRef]

32. Ouakad, H.M.; Younis, M.I. The dynamic behavior of MEMS arch resonators actuated electrically. Int. J. Non Linear Mech. 2010, 45, 704-713. [CrossRef]

33. Kacem, N.; Baguet, S.; Hentz, S.; Dufour, R. Computational and quasi-analytical models for non-linear vibrations of resonant MEMS and NEMS sensors. Int. J. Non Linear Mech. 2011, 46, 532-542. [CrossRef]

34. Souayeh, S.; Kacem, N. Computational models for large amplitude nonlinear vibrations of electrostatically actuated carbon nanotube-based mass sensors. Sens. Actuators A Phys. 2014, 208, 10-20. [CrossRef]

(C) 2016 by the authors; licensee MDPI, Basel, Switzerland. This article is an open access article distributed under the terms and conditions of the Creative Commons Attribution (CC-BY) license (http://creativecommons.org/licenses/by/4.0/). 